\title{
Awareness of Diabetic Foot Disease and Practice of Foot Care among Patients with Diabetes Mellitus in Tertiary Healthcare Centers
}

\author{
Kaladi Anjinappa Suhasini ${ }^{1}$, Shashikala Manjunatha ${ }^{2}$, Basavaraju Santosh ${ }^{3}$, Channaveeradevaru Chandrakala ${ }^{4}$
}

\begin{abstract}
Introduction: The prevalence of diabetic foot in diabetes mellitus (DM) patients has increased enormously. Diabetic foot disease (DFD) is one of the debilitating complications caused by DM and is the leading cause of non-traumatic lower limb amputation. The majority of diabetic patients are not properly educated about foot disease and foot care. In this study, we tried to assess the awareness about foot-related problems among patients with DM visiting tertiary healthcare centers. We also assessed the practice of foot care among patients with diabetes.

Materials and methods: This cross-sectional study was carried out in patients with DM attending tertiary healthcare centers after Ethical committee approval. The study was conducted in outpatient departments of endocrinology at tertiary care hospitals, in Bengaluru, using a predefined questionnaire. The results were analyzed using appropriate statistical methods.

Results: One hundred and twenty-three patients with DM were recruited and given the questionnaire. The mean age of patients was 52.57 \pm 12.45 years and the mean duration of diabetes was $11.7 \pm 8.63$ years. There were 40 male patients $(32.5 \%)$ and 83 female patients (67.5\%). Ninety people were from the urban (73.17\%) and the remaining 33 people were from the rural area (26.83\%). Out of 123 , only 102 patients (82.5\%) were aware that diabetes causes foot problems. Though most of them (72.5\%) knew that uncontrolled diabetes can cause wound/ ulcer, and $33 \%$ were aware that uncontrolled diabetes can lead to gangrene. Only $15 \%$ of patients were aware that DFD can lead to lower limb amputation. Forty-six patients (37.5\%) were aware that smoking increases the incidence of DFD and 58 (47.5\%) know that previous foot disease can increase the risk of foot problems in the future. Forty-three patients (35\%) were aware that the presence of foot problems increases the risk of heart disease in diabetic patients. Eighty-six subjects (70\%) were aware that there is specially customized footwear available for diabetic patients. One hundred and ten subjects (89.4\%) were aware that good glycemic control from the beginning helps prevent diabetes-related foot problems. Only 49 subjects (39.8\%) were taking care of their feet, of which only 18 patients examined and took care of the feet daily. About 120 people in the study used footwear outdoor (97.5\%) and only 23 people (18.6\%) used footwear both outside and inside the house. Three people in the study did not use footwear even outside the house.

Conclusion: It is vital to educate the patients about foot disease and foot care as a part of diabetes management. By improving awareness, we can decrease the occurrences of foot disease, improve the quality of life, and promote the overall health of a diabetic individual.

Keywords: Diabetes mellitus, Diabetic foot disease, Foot care.

The Journal of Medical Sciences (2020): 10.5005/jp-journals-10045-00149
\end{abstract}

\section{INTRODUCTION}

Diabetes mellitus (DM) is one of the common conditions that we come across in our practice. About 463 million people in the world had DM in 2019, and it is estimated that 700 million people will have the disease by 2045 . By 2019, India had 77 million people living with diabetes and it is expected to increase to 134.2 million by 2045 . More importantly, about $50 \%$ of people with DM are undiagnosed. ${ }^{1}$

Uncontrolled diabetic status is associated with multiple microvascular and macrovascular complications. Of these, one of the important and distressing complications many diabetic patients suffer is the occurrence of diabetic foot disease (DFD). ${ }^{2}$ The pathophysiology of DFD involves destructive changes in the nerves and vessels of the lower limbs, which are the direct result of poorly controlled DM. This leads to local tissue destruction as a result of sensory/motor neuropathy and compromised blood flow to the lower extremities, causing various manifestations of DFD like wound, ulcer, gangrene, etc., in diabetic patients. These risk factors like neuropathy and vasculopathy are present in $>10 \%$ of patients at the time of diagnosis of type 2 diabetes mellitus (T2DM). Diabetic foot disease is one of the major challenges to the healthcare system in both developed and developing countries, with substantial economic consequences for the patients, their \begin{tabular}{l}
\hline 1,2,4 Department of Community Medicine, RajaRajeswari Medical \\
College and Hospital, Bengaluru, Karnataka, India \\
${ }^{3}$ Department of Endocrinology, Sagar Hospitals, Bengaluru, Karnataka,
\end{tabular} India

Corresponding Author: Kaladi Anjinappa Suhasini, Department of Community Medicine, RajaRajeswari Medical College and Hospital, Bengaluru, Karnataka, India, Phone: +91 8074230385, e-mail: suhasini. rrmch@gmail.com

How to cite this article: Suhasini KA, Manjunatha S, Santosh B, et al. Awareness of Diabetic Foot Disease and Practice of Foot Care among Patients with Diabetes Mellitus in Tertiary Healthcare Centers. J Med Sci 2020;6(2):36-39.

Source of support: Nil

Conflict of interest: None

families, and society. Diabetic foot disease is known to account for $20 \%$ of all hospitalizations of T2DM patients, and it also leads to chronic disabilities, lower limb amputation, and death, apart from causing economic burden. ${ }^{2,3}$ One of the studies showed that the risk of amputation is increased by 12.3 times in diabetic patients as compared to the normal population. ${ }^{4}$ 
The United Kingdom Prospective Diabetes Study (UKPDS) has shown that early and intensive control of blood glucose through adequate diet, exercise, and medications will help in preventing the development of micro- and macro-vascular complications in diabetic patients. ${ }^{5}$ It is also important to follow good practice of diabetic foot care such as daily foot examination and use of appropriate footwear, which will help in early detection of DFD and prevent its progression and development of further complications. People with poor knowledge and improper practice of diabetic foot care are known to be at a higher risk of diabetic foot ulcers. ${ }^{6}$ Simple health education measures can improve both the knowledge and practice of diabetic foot care in diabetic patients. ${ }^{7}$ Adoption of foot care practice has also been shown to reduce foot problems such as corns and callosities and promote healing of foot ulcers. ${ }^{8}$ However, in India, there is a paucity of studies that have focused on assessing the knowledge and practice of diabetic foot care in diabetic patients. Thus, we conducted this study to assess the knowledge regarding DFD and the practice of foot care in diabetic patients in tertiary care centers.

\section{Materials and Methods}

This is an observational descriptive cross-sectional study performed between July 2019 and September 2019 on an outpatient population attending Endocrinology OPD at a tertiary care hospital and a corporate hospital in Bengaluru, India. Both the hospitals cater to urban and rural populations. The Ethical approval to conduct the study was taken from the Biomedical Research Ethics Committee of both the institutes.

Through the pilot study, the proportion of diabetic foot awareness was found to be $p=25 / 30=0.83$. Then, the sample size was calculated based on Krejcie-Morgan formula, after consultation with the biostatistician. $n=\chi^{2} \mathrm{~N} \mathrm{P}(1-\mathrm{P}) / \mathrm{e}(\mathrm{N}-1)+\chi^{2} \mathrm{P}(1-\mathrm{P}){ }^{9}$ One hundred and twenty-three patients were included in the study. The inclusion criteria were T2DM patients, age $\geq 18$ years, had been on treatment for $\geq 12$ months, and have visited the diabetic clinic at our hospitals. Patients who did not give consent to participate in the study, T1DM patients, those who were cognitively impaired, and those who had debilitating illnesses were excluded from the study. A systematic randomized sampling method was applied to select participants. Every third patient meeting the inclusion criteria were included in the study. Precautions were taken to ensure the participants did not participate in the study more than once.

Patients' demographic data were collected. Variables such as age, gender, race, and the duration of DM were noted along with the educational level and household income per month.

A validated, semi-structured, predetermined questionnaire was prepared. It was prepared in English and then translated to the local language Kannada. Signed written informed consent was taken from every participant who was willing to participate in the study. The questionnaire and an information sheet were distributed to each participant. The responses to the questionnaire were filled anonymously to protect the confidentiality of all the participants. The data were captured on an Excel spreadsheet and then statistically analyzed using IBM SPSS version 23 .

\section{Results}

One hundred and twenty-three participants who met the inclusion criteria and gave informed consent were included in the study. They received the questionnaire, the demographic data collected, and the responses to the questions were noted. The average age of the study population was $52.57 \pm 12.45$ years. Of the study population, $40(32.5 \%)$ were male and $83(67.5 \%)$ were female. The mean duration of diabetes was $11.7 \pm 8.63$ years. Ninety people $(73.17 \%)$ were from urban backgrounds while the remaining 33 (26.83\%) were belonging to rural areas.

About 102 out of 123 patients with T2DM (82.5\%) were aware uncontrolled diabetes can lead to DFD. Among these, $72.5 \%$ of patients knew uncontrolled diabetes can cause infection and ulcers, while only $33 \%$ knew it can lead to gangrene and $15 \%$ knew it may lead to amputation of lower limb extremities. Only about 46 members in the study (37.39\%) were aware that smoking can increase the incidence of foot problems in diabetic patients. Among the study population, 58 (47.1\%) patients were aware that the presence of previous foot problems in diabetics will increase the recurrence of foot problems in the future. Only 43 patients $(34.9 \%)$ in the study knew that the presence of DFD is associated with a higher risk of coronary heart disease. About 110 members in the study (89.4\%) were aware that adequate management of diabetes with diet and lifestyle modifications and appropriate medicines will prevent the development of foot problems. Eighty-six patients (69.9\%) knew that there is specially customized footwear available for diabetic patients.

About 49 (39.8\%) out of 123 patients were taking care of their feet by themselves while the remaining 74 (60.2\%) were not. Among them, only 18 patients were examining their feet every day, another 18 did their feet examination once a week, and the remaining 13 did it about once a month. One hundred and twenty patients (97.5\%) used footwear outside the house and 23 patients (18.6\%) used footwear both inside and outside their house. However, three patients did not use footwear even while walking outside the house (Table 1).

\section{Discussion}

Our study showed that although most of the diabetic patients included in the study were aware that uncontrolled diabetes can lead to foot problems, most of them were not aware of all the complications of DFD. These results were consistent with the results of some of the similar studies done in India as well as other countries. ${ }^{10-13}$ About $82.5 \%$ of our study population were aware uncontrolled diabetes can lead to DFD. Among these, $72.5 \%$ of patients knew uncontrolled diabetes can cause infection and ulcer, while only $33 \%$ knew it can lead to gangrene and $15 \%$ knew it may lead to amputation of lower limb extremities. A study conducted in India by Taksande et al. in 2017 showed that only $23 \%$ of the study population was aware of DFD and its complications. ${ }^{10}$ A study done in South Africa by Goie and Naidoo in 2016 showed that about $59.6 \%$ of diabetic patients in the study were aware that uncontrolled diabetes can cause ulcers and about $32.9 \%$ of people knew it could lead to gangrene. ${ }^{11}$ Another study done in India by Saurabh et al. in 2014 found that only about $54.4 \%$ of the diabetic population in the study were aware that diabetes can lead to the ulcer of the feet. ${ }^{12}$ Our study showed that comparatively more people with diabetes were aware of DFD compared to some of these studies. The reason for this difference could be due to the higher percentage of the urban population in our study $(73.17 \%)$ and hence responders were more educated and aware, while the above two Indian studies were done on the rural population. ${ }^{10,12}$

Only about 46 members in the study (37.39\%) were aware that smoking can increase the incidence of foot problems in diabetic 
Table 1: Factors associated with knowledge and practice of diabetic foot care

\begin{tabular}{|c|c|c|c|c|}
\hline \multicolumn{2}{|c|}{ Related to awareness } & \multirow{2}{*}{$\frac{\text { Yes }}{102(82.5 \%)}$} & \multirow{2}{*}{$\frac{\text { No }}{12(10 \%)}$} & \multirow{2}{*}{$\begin{array}{l}\text { Don't know } \\
9(7.5 \%)\end{array}$} \\
\hline I & Can diabetes cause foot problems? & & & \\
\hline II & Does smoking increase diabetic foot problems? & $46(37.5 \%)$ & $31(25 \%)$ & $46(37.5 \%)$ \\
\hline III & $\begin{array}{l}\text { Does previous } \mathrm{h} / \mathrm{o} \text { foot disease increase the risk of } \\
\text { recurrent diabetic foot disease? }\end{array}$ & $58(47.1 \%)$ & $18(15 \%)$ & $47(37.9 \%)$ \\
\hline IV & $\begin{array}{l}\text { Does diabetic foot disease increase the risk of heart } \\
\text { disease? }\end{array}$ & $43(34.9 \%)$ & $34(27.6 \%)$ & $46(37.5 \%)$ \\
\hline V & $\begin{array}{l}\text { Are there customized footwear available for diabetic } \\
\text { patients? }\end{array}$ & $86(70 \%)$ & $28(22.5 \%)$ & $9(7.5 \%)$ \\
\hline VI & $\begin{array}{l}\text { Does good glycemic control prevent diabetic foot } \\
\text { problems? }\end{array}$ & $110(89.4 \%)$ & $8(6.5 \%)$ & $5(4.1 \%)$ \\
\hline \multicolumn{5}{|c|}{ Related to practice } \\
\hline I & Are you taking care of your feet? & Yes: 49 (39.8\%) & No: $74(60.2 \%)$ & \\
\hline II & $\begin{array}{l}\text { How often do you examine your foot and footwear? (out } \\
\text { of } 49 \text { people) }\end{array}$ & Daily: 18 (36.7\%) & Weekly: 18 (36.7\%) & $\begin{array}{l}\text { Monthly: } 13 \\
(26.6 \%)\end{array}$ \\
\hline III & Do you use footwear? & $\begin{array}{l}\text { Outside the house } \\
\text { only: } 120(97.5 \%)\end{array}$ & $\begin{array}{l}\text { Outside and inside the } \\
\text { house: } 23(18.6 \%)\end{array}$ & \\
\hline
\end{tabular}

patients. A study by Goie and Naidoo showed that about $62.5 \%$ of diabetic patients in a similar study were aware that smoking is associated with reduced blood flow to the lower limbs and can lead to DFD. ${ }^{11}$ Among our study population, only 58 (47.1\%) patients were aware that the presence of previous foot problems in diabetics will increase the recurrence of foot problems in the future. Hence, the proper knowledge was lacking in most of our study population.

Only 43 patients (34.9\%) in the study knew that the presence of DFD is associated with a higher risk of coronary heart disease. One of the causes for DFD is peripheral vascular disease and it shares the pathophysiology with coronary artery disease. ${ }^{14}$ However, many patients in the study were not aware regarding increased coronary artery disease risk in those with DFD. It is important to educate diabetic patients about this aspect and treat them appropriately to prevent both complications.

About 110 members in our study (89.4\%) were aware that adequate management of diabetes with diet and lifestyle modifications and appropriate medicines will prevent the development of foot problems. A Malaysian study done by Mohammad-Lutfi et al. in 2014 showed that $93.6 \%$ of the study population were having the knowledge that adequate diabetes management prevents foot problems. ${ }^{13}$ A similar study done in India by Saurabh et al. in 2014 found that $91.1 \%$ of the study population were aware that good glycemic control helps in preventing diabetic foot complications. ${ }^{12}$ A study done in South Africa by Goie and Naidoo in 2016 showed that $88.6 \%$ of the study population were aware that good diabetes management and adequate glucose control helps in preventing foot problems in diabetic patients. ${ }^{11}$ The results of our study were consistent with the results of other studies regarding the knowledge among diabetic people.

Eighty-six people (69.9\%) in our study knew that there is specially customized footwear available for diabetic patients. A similar Malaysian study done by Mohammad-Lufti et al. in 2014 showed that about $70 \%$ of their study population were aware of specially customized footwear for diabetic patients. ${ }^{13}$ Our study results of awareness of specialized footwear are similar to this study.

About 49 (39.8\%) out of 123 patients in our study were taking care of their feet by themselves while the remaining 74 (60.2\%) were not. Among these 49 patients, only 18 were examining their feet every day, another 18 did their feet examination once a week, and the remaining 13 did it about once a month. In a Malaysian study done by Mohammad-Lufti et al. in 2014, about $50.7 \%$ of diabetic patients inspected their feet..$^{13}$ A South African study by Goie and Naidoo in 2016 found that $65 \%$ of the study population did examine their feet every day. ${ }^{11}$ In an Indian study by Saurabh et al., about $52.4 \%$ examined their feet. ${ }^{12}$ Another Indian study by Taksande et al. conducted on rural diabetic population found that none of the study members did examine their feet. ${ }^{10}$ Most Indian diabetic patients are not educated and not aware of daily foot examination.

In our study, 120 people (97.5\%) used footwear outside the house and 23 people (18.6\%) used footwear both inside and outside the house. However, three patients did not use footwear even while walking outside the house. A Malaysian study done by MohammadLufti et al. in 2014 showed that about $77.1 \%$ of study people used footwear. ${ }^{13}$ In an Indian study by Saurabh et al. in 2014 done in the rural diabetic population, about $97.1 \%$ of patients used footwear outside the house and only $7.6 \%$ of patients in the study used footwear both outside and inside the house. ${ }^{12}$ The comparatively higher percentage of patients in our study using footwear inside the house could be due to the inclusion of both rural and urban people in our study.

\section{ConClusion}

As seen in our study, not all diabetic patients are aware of DFD and its complications. So all diabetic patients must be counseled about the associated foot problems and educated about regular foot self-examination and appropriate foot care, during every hospital visit. By improving awareness, we can decrease the occurrences of foot disease, improve the quality of life, and promote the overall health of a diabetic individual. Hence, proper education of diabetic patients at the primary care level is very important for reducing the burden of its complications.

\section{References}

1. International Diabetes Federation. IDF Diabetes Atlas. 9th ed., Brussels, Belgium 2019. Available at: https://www.diabetesatlas.org.

2. Boulton A, Vileikyte L, Ragnarson-Tennvall G, et al. The global burden of diabetic foot disease. Lancet 2005;366:1719-1724. 
3. Beers MH, Porter RS, Jones TV, et al., ed. Diabetes mellitus and disorders of carbohydrate metabolism. The Merck Manual. 18th ed., New Jersey, USA: Merck Research Laboratories; 2006. pp. 1274-1294.

4. Morris $A D$, McAlpine $R$, Steinke $D$, et al. Diabetes and lower-limb amputations in the community. A retrospective cohort study. DARTS/ MEMO Collaboration. Diabetes audit and research in Tayside Scotland/ medicines monitoring unit. Diabetes Care 1998;21(5):738-743.

5. Intensive blood-glucose control with sulphonylureas or insulin compared with conventional treatment and risk of complications in patients with type 2 diabetes (UKPDS 33). Lancet 1998;352:837-853.

6. Chellan G, Srikumar S, Varma AK, et al. Foot care practice-the key to prevent diabetic foot ulcers in India. Foot (Edinb) 2012;22:298-302.

7. Vatankhah N, Khamseh ME, Noudeh YJ, et al. The effectiveness of foot care education on people with type 2 diabetes in Tehran, Iran. Prim Care Diabetes 2009;3:73-77.

8. Viswanathan V, Madhavan S, Rajasekar S, et al. Amputation prevention initiative in South India: positive impact of foot care education. Diabetes Care 2005;28:1019-1021.
9. Krejcie RV, Morgan DW. Determining sample size for research activities. Educ Psychol Meas 1970;30:607-610.

10. Taksande BA, Thote M, Jajoo UN. Knowledge, attitude, and practice of foot care in patients with diabetes at central rural India. J Family Med Prim Care 2017;6:284-287.

11. Goie TT, Naidoo M. Awareness of diabetic foot disease amongst patients with type 2 diabetes mellitus attending the chronic outpatients department at a regional hospital in Durban, South Africa. Afr J Prm Health Care Fam Med 2016;8(1):1170.

12. Saurabh S, Sarkar S, Selvaraj K, et al. Effectiveness of foot care education among people with type 2 diabetes in rural Puducherry, India. Indian J Endocrinol Metab 2014;18(1):106-110.

13. Muhammad-Lutfi AR, et al. Knowledge and practice of diabetic foot care in an in-patient setting at a tertiary medical center. Malaysian Orthopae J 2014;8(3):22-26.

14. Kannel WB, Wilson PW. An update on coronary risk factors. Med Clin North America 1995;75:951-971. 